\title{
Cardiac resynchronization therapy coronary venous left ventricular lead removal and reimplantation: Experience from a single center in China
}

\author{
WENCHENG ZHENG, DING LI, XUEBIN LI, FENG ZE, LONG WANG, \\ JIANGBO DUAN and CUIZHEN YUAN \\ Department of Cardiac Electrophysiology, Peking University People's Hospital, \\ Peking University, Beijing 100044, P.R. China
}

Received November 12, 2018; Accepted June 27, 2019

DOI: $10.3892 /$ etm.2019.7818

\begin{abstract}
The present study aimed to investigate the success rate, methods and associated complications of left ventricular lead (LVL) extraction and venous pathway reimplantation in patients with cardiac resynchronization therapy device/defibrillator (CRT/CRTD). A retrospective analysis was performed in the patients who underwent CRT/CRTD extraction and reimplantation at our hospital from January 2012 to October 2018. The methods, patient complications and success rate of extraction and reimplantation of LVL were analyzed. A total of 54 patients underwent CRT/CRTD removal (pacemaker infection, $n=51$; LVL dysfunction, $n=3$; CRT/CRTD, 34/20). A total of 54 LVLs were removed (3 active electrodes and 51 passive electrodes). The average implantation duration of the LVL was 53.5 months (range, 1-204 months), whereas the success rate of the LVL extraction was $100 \%$ (94\% completely removed and $6 \%$ clinically removed. A total of 6 patients $(11 \%)$ were treated only by manual traction, whereas the remaining patients had their LVL successfully removed using extraction tools. In the peri-operative period, one fatality occurred (2\%). The highest complication rate of the lead extraction was $2 \%$ and no minor complications were observed. A total of 36 patients were reimplanted on the right side, which was successful in 31 cases (success rate, 86.1\%), whereas 3 cases were successfully reimplanted on the left side. The total success rate of LVL reimplantation was $87.2 \%$. The procedure of the LVL removal and reimplantation exhibited a high success rate and
\end{abstract}

Correspondence to: Dr Ding Li, Department of Cardiac Electrophysiology, Peking University People's Hospital, Peking University, 11 Xizhimen South Street, Xicheng, Beijing 100044, P.R. China

E-mail: lidingpku666@126.com

Key words: cardiac resynchronization therapy, left ventricular lead removal, reimplantation after extraction a lower incidence of complications compared with that in patients with cardiac devices.

\section{Introduction}

The increasing use of cardiac resynchronization therapy device/defibrillators (CRT/CRTDs) has been paralleled by a rise in the requirement for CRT/CRTD extraction and reimplantation due to device infection or dysfunction. The CRT/CRTD system, which is notably used for left ventricular lead (LVL) removal, appears to be associated with a higher risk than conventional pacemakers due to the thin wall of the coronary sinus (CS) and superior vena cava system. CRT/CRTD devices are usually implanted via the left subclavian vein. The right subclavian vein is used to reimplant the CRT/CRTD system following the removal of infected devices. However, the stenosis/occlusion of the original CS branch and the anatomical complexity of the right-side pathway render reimplantation considerably difficult. To date, studies on LVL removal and reimplantation have been limited to small sample size studies (1-4), whereas no previous studies have examined these parameters in Chinese patients, to the best of our knowledge. The present study was performed in the largest removal and reimplantation center for CRT/CRTD in China and its aim was to provide information on the success rate and incidence of complications in patients with $\mathrm{LVL}$ removal and reimplantation.

\section{Materials and methods}

Patients. All patients with CRT/CRTD who underwent device removal due to infection or dysfunction between January 2012 and October 2018 were enrolled. The present study was approved by the Ethics Committee of Peking University People's Hospital (Beijing, China). All patients enrolled provided written informed consent for their participation in the study and for publication of associated images. The patients' characteristics, as well as laboratory blood test and imaging results, were analyzed. The indications of lead extraction were determined based on the 2017 Heart Rhythm Society (HRS) expert consensus statement on cardiovascular 
implantable electronic device lead management and extraction (5). High-risk patients included a duration of passive fixed lead implantation of $>10$ years, a duration of defibrillation lead implantation of $>3$ years, a duration of lead perforation of $>1$ month and a total number of electrodes of $>5$.

Electrode extraction. The lead extraction methods were performed as previously described $(4,6)$. The lead was removed under general anesthesia and transesophageal ultrasound monitoring in high-risk patients, and the remaining patients were subjected to local anesthesia. Pacemaker-dependent patients were implanted with a right ventricular (RV) pacing lead through the left femoral vein. Bilateral elbow venography was performed prior to extraction. The lead devices were removed using a manual or locking stylet traction in case where were implanted 12 months prior to enrolment (Cook Medical).

The femoral vein approach (Cook Medical) was used by an evolution mechanical sheath (Cook Medical) and/or a laser sheath (CVX-300). These methods were applied alone or in combination in those patients with an implantation duration of $>12$ months. Endocardial active fixation ventricular electrodes were implanted in the pacemaker-dependent patients as a transition to permanent pacemaker reimplantation. The definitions of successful removal or clinical success of removal in the presence or absence of complications were according to the 2017 HRS expert consensus (5).

Reimplantation of LV electrode. The reimplantations were performed on the contralateral side in infective patients (all via the right subclavian vein). The LV lead dysfunction was reimplanted at the ipsilateral side. Antimicrobial therapy according to the 2017 HRS expert consensus recommendations was used for the management of the suspected electronic device-associated infection. The antimicrobial therapy used for endocarditis has a minimum duration of 4-6 weeks, whereas for bacteremia, it has a lower duration (at least 2 weeks) (5). A new implantation may be reasonably postponed until blood cultures are negative for $72 \mathrm{~h}$ in patients with bacteremia and endocarditis (5). In subjects with pocket site infection, the reimplantation procedure was performed with a treatment duration of 48-72 $\mathrm{h}$ following device removal (5). Optional balloon dilatation was used if the target vein exhibited apparent stenosis. RV double site or bundle pacing were performed for patients with LVL reimplantation failure.

Statistical analysis. Continuous variables are expressed as the mean \pm standard deviation for parametric variables. Dichotomous variables were presented as an absolute number with percentage. All analyses were performed using SPSS software (version 18.0; SPSS Inc.).

\section{Results}

Case characteristics. A total of 54 patients with infection/dysfunction $(n=51 / 3)$ were enrolled (CRT/CRTD, 34/20). The general information of these patients is presented in Table I. The average age of patients was $65.33 \pm 7.38$ (male, 46; female, 8). As presented in Table II, a total of 41 patients (76\%) exhibited pocket infection, 7 (13\%) presented with bacteremia and $3(6 \%)$ with infective endocarditis. The dimensions of the
Table I. Baseline characteristics of the patients ( $\mathrm{n}=54)$.

\begin{tabular}{lc}
\hline Parameter & Value \\
\hline Age (years) & $65.33 \pm 7.38$ \\
Gender (male) & $46(85)$ \\
CRT/CRTD device & $34 / 20$ \\
Ischemic cardiomyopathy & $11(20)$ \\
Atrial fibrillation & $6(11)$ \\
Serum white blood cell count $/ 1$ & $8.72 \pm 3.07 \times 10^{9}$ \\
Serum hemoglobin g/l & $123.39 \pm 13.17$ \\
Serum creatinine $\mu$ mol/l & $86.73 \pm 30.85$ \\
Hypertension & $22(41)$ \\
Hyperlipidemia & $8(15)$ \\
History of CVA or TIA & $12(22)$ \\
History of CABG & $3(6)$ \\
Diabetes mellitus & $8(15)$ \\
Left ventricular ejection fraction $(\%)$ & $33.17 \pm 9.86$ \\
Left ventricular end-diastolic diameter $(\mathrm{mm})$ & $67.77 \pm 9.81$ \\
Left ventricular end-systolic diameter $(\mathrm{mm})$ & $46.75 \pm 10.39$ \\
\hline
\end{tabular}

Values are expressed as $\mathrm{n}(\%)$ or the mean \pm standard deviation. CVA, cerebrovascular accident; TIA, transient ischemic attack; CABG, coronary artery bypass grafting; CRTD, cardiac resynchronization therapy defibrillator.

Table II. Types of pacemaker and electrode infection and implantation details in the total cohort $(\mathrm{n}=54)$.

\begin{tabular}{lc}
\hline Feature & Value \\
\hline Pocket infection & $41(76)$ \\
Bacteremia & $7(13)$ \\
Infective endocarditis & $3(6)$ \\
Vegetation (transverse diameter x & \\
vertical diameter cm) & \\
Left ventricular lead dysfunction & $1.3 \times 1.5$, \\
& $1.5 \times 2.1$, \\
& $1.6 \times 1.7$ \\
& $3(6)$ \\
Total number of leads removed & 156 \\
Average number of leads removed per case & 2.95 \\
Average time of lead implantation (months) & 53.5 \\
Special left ventricular leads & $3^{\mathrm{a}}$ \\
\hline
\end{tabular}

${ }^{\text {aMedtronic }} 4195$ Starfix active fixation lead. Values are expressed as $\mathrm{n}$ or $\mathrm{n}(\%)$ unless otherwise specified.

vegetation were $1.3 \times 1.5 \mathrm{~cm}, 1.5 \times 2.1 \mathrm{~cm}$ and $1.6 \times 1.7 \mathrm{~cm}$ (transverse diameter $x$ vertical diameter $\mathrm{cm}$ ), respectively. Following infection, 29 (54\%) of the patients had failed debridement at local hospitals. In the 54 patients, 156 electrode leads were removed and the average implantation time was 53.5 months. Among these patients, a Medtronic 4195 Starfix LV active lead was used in 3 cases (Table II). In addition, 3 non-infectious 
Table III. Extraction of LVL and associated complications in the cohort $(n=54)$.

\begin{tabular}{lc}
\hline Extraction method or complication & N $(\%)$ \\
\hline LVLremoval complete success & $51(94)$ \\
LVL removal clinical success & $3(6)$ \\
Bare-handed extraction & $6(11)$ \\
Locking steel wire extraction & $23(43)$ \\
Locking steel wire combined with & $1(2)$ \\
evolution sheath extraction & \\
Snare catcher extraction & $24(44)$ \\
Patient death after LVL extraction & $1(2)$ \\
Major complications & $1(2)$ \\
Pericardium tamponade & $0(0)$ \\
Respiratory failure & $1(2)$ \\
In-cath lab deaths & $0(0)$ \\
Minor complications & $0(0)$ \\
Hematoma requiring evacuation & $0(0)$ \\
Significant blood loss requiring blood transfusion & $0(0)$
\end{tabular}

LVL, left ventricular lead.

Table IV. Details regarding removal and reimplantation of RA and $\mathrm{RV}$ in patients $\left(\mathrm{n}=51^{\mathrm{a}}\right)$.

\begin{tabular}{|c|c|}
\hline Item & $\mathrm{N}(\%)$ \\
\hline RA and RV lead removal complete success & $49(96.1)$ \\
\hline RA and RV lead removal clinical success & $2(3.9)$ \\
\hline Total number of RA leads extracted & 51 \\
\hline Total number of RV defibrillation leads extracted & 17 \\
\hline Total number of RV general leads extracted & 34 \\
\hline $\begin{array}{l}\text { Reimplantation CRTD to the right after } \\
\text { original extraction }\end{array}$ & 8 \\
\hline $\begin{array}{l}\text { Reimplantation CRT to the right after original } \\
\text { CRTD extraction }\end{array}$ & 7 \\
\hline $\begin{array}{l}\text { Cases requiring laser extraction of RV } \\
\text { defibrillation leads }\end{array}$ & $3(5.9)$ \\
\hline Cases requiring Snare extraction & $31(57.4)$ \\
\hline \multicolumn{2}{|c|}{$\begin{array}{l}{ }^{\text {a }} \text { eft ventricular lead dysfunction and non-infectious RA and RV } \\
\text { leads retained in three cases, leading to a total number of } 51 \text {. RA, } \\
\text { right atrial; RV, right ventricular; CRTD, cardiac resynchronization } \\
\text { therapy defibrillator. }\end{array}$} \\
\hline
\end{tabular}

patients underwent electrode extraction and reimplantation due to elevated LV pacing threshold and severe diaphragmatic stimulation.

Electrode wire removal and associated complications. The success rate of complete and clinical removal of the LV electrodes was 94 and 6\%, respectively. Manual traction was used in 6 patients $(11 \%)$, whereas removal with locking of steel wire was performed in 23 patients $(43 \%)$. In one patient
Table V. Details regarding reimplantation of LVL.

\begin{tabular}{lcc}
\hline Item & $\begin{array}{c}\text { Cases, } \\
\mathrm{n}(\%)\end{array}$ & $\begin{array}{c}\text { Success } \\
\text { rate (\%) }\end{array}$ \\
\hline $\begin{array}{l}\text { Reimplant on right side of LVL } \\
\text { Successful reimplantation of LVL } \\
\text { on the right side }\end{array}$ & 36 & 86.1 \\
$\begin{array}{l}\text { Successful reimplantation of LV } \\
\text { dysfunction lead on left side }\end{array}$ & 3 & \\
$\begin{array}{l}\text { Total cases of successful } \\
\text { reimplantation after extraction }\end{array}$ & 34 & \\
$\begin{array}{l}\text { Patient refused reimplantation } \\
\text { Biventricular pacing deemed to } \\
\text { be no longer indicated }\end{array}$ & $7(12.9)$ & \\
$\begin{array}{l}\text { Deemed to be high risk } \\
\text { for reimplantation }\end{array}$ & $4(6)$ & \\
Patient death prior to reimplantation & $1(2)$ & \\
\hline \begin{tabular}{l} 
LVL, left ventricular lead. \\
\hline
\end{tabular} & & \\
\hline
\end{tabular}

Table VI. Details regarding coronary venography and reimplantation site of LVL in the relevant cases $\left(n=39^{a}\right)$.

\begin{tabular}{lc}
\hline Item & $\mathrm{N}(\%)$ \\
\hline $\begin{array}{l}\text { Original lateral vein stenosis }>50 \% \\
\text { after LVL extraction }\end{array}$ & $16(41.0)$ \\
$\begin{array}{l}\text { Original lateral vein occluded after LVL extraction } \\
\text { Reimplantation into original lateral vein }\end{array}$ & $3(7.7)$ \\
$\begin{array}{l}\text { after LVL extraction } \\
\text { Reimplantation in other lateral vein due to } \\
\text { original stenosis or occlusion } \\
\text { Balloon used to dilate original lateral vein stenosis }\end{array}$ & $10(25.6)$ \\
\end{tabular}

${ }^{a}$ Total cases of LVL reimplantation, $n=39$. LVL, left ventricular lead.

(2\%), a lock wire combined with evolution sheath was used. In a total of 24 cases (44\%) the electrodes were removed by Snares via the femoral vein (Table III; Fig. 1C). The success rate of complete removal of the right atrial and RV electrodes was $96.1 \%$, whereas the success rate of clinical removal was $3.9 \%$ (Table IV). Following LVL extraction, one patient did not survive (mortality rate, $2 \%$ ) due to severe heart failure. No minor complications were observed in the cohort.

Reimplantation of LV electrode and CRT/CRTD. A total of 3 out of 39 cases underwent LVL reimplantation (right/left side, 36/3). In a total of 31 cases, right LVL reimplantation was successful, and all 3 left LVL reimplantations were successful. The total success rate of LVL reimplantation was $87.2 \%$. This percentage was decreased to $86.1 \%$ for the right-side approach (Fig. 2; Table V). After LVL extraction, stenosis of the original lateral vein was $>50 \%$ in 16 cases $(41.0 \%)$ and occlusion of the original lateral vein occurred in 3 cases $(7.7 \%$; Table VI). Severe stenosis of the original posterior vein was observed 

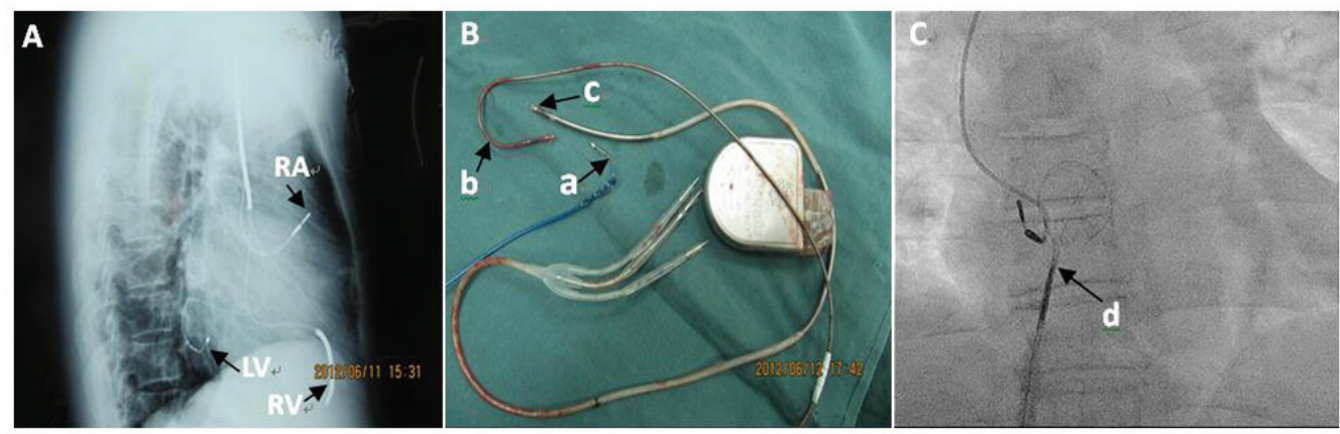

Figure 1. (A) Horizontal position angle X-ray imaging, displaying the device relative to the location of the RA, LV and RV. (B) Device after extraction; a, LV active fixing lead and its external blue insulating layer; b, RA lead; c, RV lead. (C) X-ray presenting the snare used to extract the RA lead. RA, right atrium; $\mathrm{L} / \mathrm{RV}$, left/right ventricle.

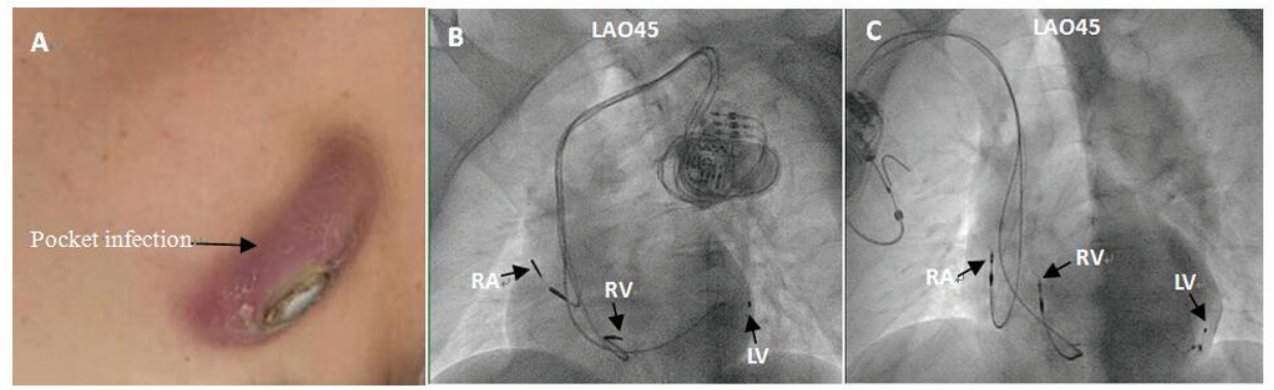

Figure 2. (A) Signal pocket infection. (B) LAO 45 angle X-ray imaging prior to CRT pacemaker extraction. (C) LAO 45 angle X-ray imaging of CRT pacemaker following right side reimplantation. LAO, left anterior oblique; CRT, cardiac resynchronization therapy; RA, right atrium; L/RV, left/right ventricle.
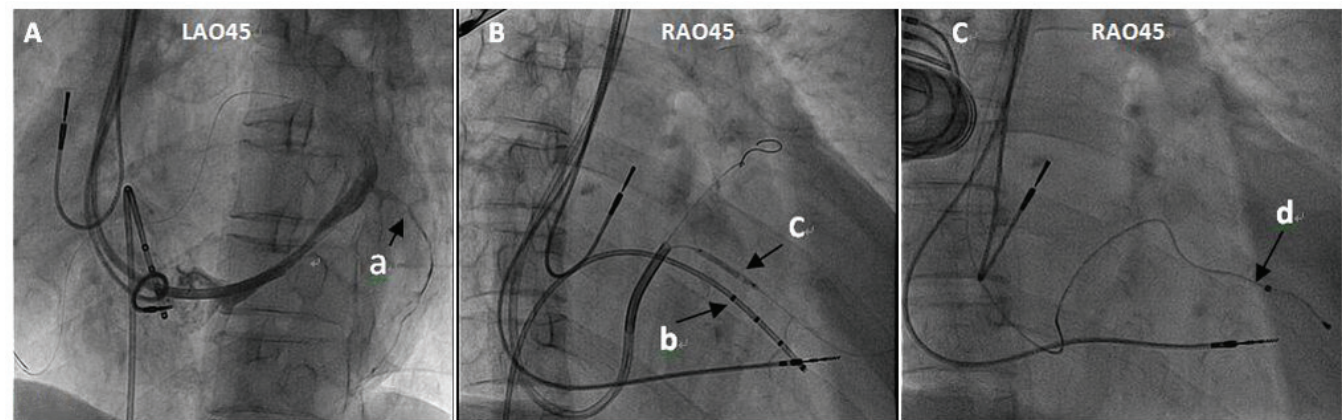

Figure 3. (A) Severe stenosis of the original lateral vein following left ventricular lead extraction on LAO 45 angle X-ray imaging (a, site of stenosis). (B) RAO 45 angle X-ray imaging. b, a $2.0 \times 20 \mathrm{~mm}$ balloon was used to dilate the original lateral vein stenosis at 6-8 atmospheric pressure units; c, temporary pacing lead. (C) Successful reimplantation of left ventricular lead in the lateral vein following balloon dilatation in RAO 45 angle (d, reimplanted lead). LAO, left anterior oblique; $\mathrm{RAO}$, right anterior oblique.
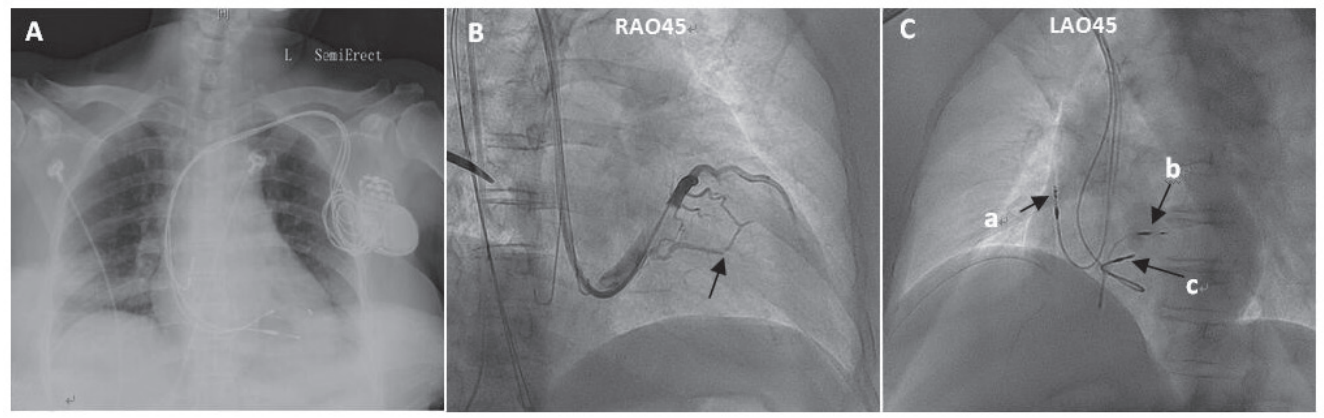

Figure 4. (A) Cardiac resynchronization therapy leads in the anteroposterior position X-ray imaging. (B) RAO 45 angle X-ray imaging displaying the original lateral vein mid-distal segment occlusion following left ventricular lead extraction (arrow). (C) LAO 45 angle X-ray imaging. Right ventricular double site pacing was selected due to original lateral vein occlusion. a, right atrial lead; b, one of the right ventricular leads; c, the remaining right ventricular lead; LAO, left anterior oblique; RAO, right anterior oblique. 
in one patient on coronary venography, and the LV lead was successfully implanted following dilatation with a 2.0x20 mm balloon at 6 or 8 atm twice (Fig. 3).

A total of 5 cases did not receive successful treatment due to severe stenosis or occlusion of the target vessel in the branch of the coronary vein. A total of 2 out of 5 patients received RV double site pacing (Fig. 4) and the other 3 patients were treated with His bundle pacing. A total of 3 non-infectious patients with LVL dysfunction were successfully reimplanted with LVL at the same stage and at the ipsilateral region following removal of the LV electrodes. A total of 7 patients refused to receive reimplantation therapy following removal of the electrodes. Following removal of the electrodes, one patient recovered to normal cardiac function and 3 patients exhibited no indication for biventricular pacing. A total of two patients exhibited atrial fibrillation with a QRS wave duration of $<120 \mathrm{msec}$ and one patient presented with persistent atrial fibrillation with a normal ejection fraction. A total of 4 patients did not receive CRT reimplantation due to their critical clinical condition (severe infection, cardiac dysfunction with severe hypotension, systemic infection and lower extremity venous thrombosis requiring anticoagulant therapy).

\section{Discussion}

The difficulties and risks of CRT/CRTD lead removal are mainly attributed to the rupture of coronary sinus and/or superior vena cava. The incidence of minor complications in this group was considerably lower than that reported in previous studies $(1,2)$. The incidence of major complications of LVL removal was $2.0 \%$ in the present study, which was consistent with that reported previously (1). The LV lead was removed successfully and safely, without the rupture of CS and of the superior vena cava due to the snare sheath's cutting effect localized to the surrounding tissue and LVL.

In the present study, the success rate of LVL reimplantation was $87.2 \%$, which was similar to that reported by a previous study (7). The majority of reimplantations was via the right subclavian vein, which is more difficult than the implantation from the left side. From the right subclavian vein to the coronary sinus, two physical curves were present, which resulted in difficulties of LVL implantation. An Amplatz L1/L2 sheath and super smooth guide wire or adjustable curve catheter may be used to guide the LVL sheath entering the coronary sinus.

The other major difficulty encountered in the reimplantion of the LVL following removal was attributed to occlusion or stenosis of the originally implanted branches. Burke et al (8) reported that the occlusion rate of the original coronary vein following removal of the LV electrode was $50 \%$. In the present study, this parameter was estimated to be $48.7 \%$. The LVL was successfully implanted in one patient with stenosis following balloon dilatation. Balloon dilatation is usually effective when the target vessel stenosis occurs during the first implantation of LVL, whereas vascular stenosis following removal frequently occurs due to fibrous tissue hyperplasia and/or thrombosis. Therefore, balloon dilatation is not recommended for routine use during reimplantation.

A total of 3 out of 5 patients who experienced unsuccessful LVL reimplantation received His bundle pacing. The clinical application of His bundle pacing has increased in recent years (9). Long-term follow-up of these cases has indicated similar results to those observed for CRT in patients with heart failure (7). This treatment may be considered in patients with CS branch occlusion following removal. However, the clinical outcome should be evaluated in a long-term follow-up period.

A total of 8 of these patients were reimplanted with CRT/CRTD on the right side following removal. Several studies have suggested that although the defibrillation threshold may be elevated in the right implantable cardiac defibrillator (ICD) compared with that noted in the left ICD, it is still considered safe and effective in clinical applications (10-12).

Taken collectively, the results of the present study suggested that CRTD/CRTD removal and reimplantation was feasible and had a relatively high success rate. However, the present study has certain limitations, including the single-center study design, the small sample size and the relatively short follow-up time period. Patients with better cardiac function and fewer complications benefit more from reimplantation within a short period of time after LVL removal. While the number of cases is limited by the low incidence, a larger cohort would provide more comprehensive and reliable information, but as our institution is the center with the largest number of cases of LVL removal in China, only these cases are available at present. According to common practice at our institution, after the LVL is removed, the patient's cardiac function and indications for CRT/CRTD are reassessed and if there is no indication, the CRT/CRTD is not reimplanted.

\section{Acknowledgements}

Not applicable.

\section{Funding}

No funding was received.

\section{Availability of data and materials}

The datasets used and/or analyzed during the present study are available from the corresponding author on reasonable request.

\section{Authors' contributions}

WZ and DL performed the study and wrote the manuscript. $\mathrm{WZ}$ and DL contributed to data analysis and interpretation. WZ, DL, XL, FZ, LW, JD and CY contributed to performing the operations.

\section{Ethics approval and consent to participate}

All experiments were performed with the approval of the Ethics Committee of Peking University People's Hospital (Beijing, China). All patients provided written informed consent regarding their participation in the study.

\section{Patient consent for publication}

Patients provided written informed consent for publication of associated images. 


\section{Competing interests}

The authors declare that they have no competing interests

\section{References}

1. Williams SE, Arujuna A, Whitaker J, Shetty AK, Bostock J, Patel N, Mobb M, Cooklin M, Gill J, Blauth C, et al: Percutaneous lead and system extraction in patients with cardiac resynchronization therapy (CRT) devices and coronary sinus leads. Pacing Clin Electrophysiol 34: 1209-1216, 2011.

2. Hamid S, Arujuna A, Khan S, Ladwiniec A, McPhail M, Bostock J, Mobb M, Patel N, Bucknall C and Rinaldi CA: Extraction of chronic pacemaker and defibrillator leads from the coronary sinus: Laser infrequently used but required. Europace 11: 213-215, 2009.

3. De Martino G, Orazi S, Bisignani G, Toscano S, Messano L, Parisi Q, Santamaria M, Pelargonio G, Dello Russo A, Bellocci F, et al: Safety and feasibility of coronary sinus left ventricular leads extraction: A preliminary report. J Interv Card Electrophysiol 13: 35-38, 2005.

4. Bongiorni MG, Zucchelli G, Soldati E, Arena G, Giannola G, Di Cori A, Lapira F, Bartoli C, Segreti L, De Lucia R and Barsotti A: Usefulness of mechanical transvenous dilation and location of areas of adherence in patients undergoing coronary sinus lead extraction. Europace 9: 69-73, 2007.

5. Kusumoto FM, Schoenfeld MH, Wilkoff BL, Berul CI, Birgersdotter-Green UM, Carrillo R, Cha YM, Clancy J, Deharo JC, Ellenbogen KA, et al: 2017 HRS Expert Consensus Statement on Cardiovascular Implantable Electronic Device Lead Management and Extraction. Heart Rhythm 14: e503-e551, 2017.
6. Bongiorni MG, Soldati E, Zucchelli G, Di Cori A, Segreti L, De Lucia R, Solarino G, Balbarini A, Marzilli M and Mariani M: Transvenous removal of pacing and implantable cardiac defibrillating leads using single sheath mechanical dilatation and multiple venous approaches: High success rate and safety in more than 2000 leads. Eur Heart J 29: 2886-2893, 2008

7. Rickard J, Tarakji K, Cronin E, Brunner MP, Jackson G, Baranowski B, Borek PP, Martin DO, Wazni O and Wilkoff BL: Cardiac venous left ventricular lead removal and reimplantation following device infection: A large single-center experience. J Cardiovasc Electrophysiol 23: 1213-1216, 2012.

8. Burke MC, Morton J, Lin AC, Tierney S, Desai A, Hong T, Kim S, Salem Y, Alberts M and Knight BP: Implications and outcome of permanent coronary sinus lead extraction and reimplantation. J Cardiovasc Electrophysiol 16: 830-837, 2010.

9. Vijayaraman P, Subzposh FA and Naperkowski A: Extraction of the permanent His bundle pacing lead: Safety outcomes and feasibility of reimplantation. Heart Rhythm 2019 (Epub ahead of print).

10. Keyser A, Hilker MK, Ucer E, Wittmann S, Schmid C and Diez C: Significance of intraoperative testing in right-sided implantable;cardioverter-defibrillators. J Cardiothorac Surg 8: 77, 2013.

11. Nery PB, Fernandes R, Nair GM, Sumner GL, Ribas CS, Menon SM, Wang X, Krahn AD, Morillo CA, Connolly SJ and Healey JS: Device-related infection among patients with pacemakers and implantable defibrillators: Incidence, risk factors, and consequences. J Cardiovasc Electrophysiol 21: 786-790, 2010.

12. Hamid S, Arujuna A, Ginks M, McPhail M, Patel N, Bucknall C and Rinaldi C: Pacemaker and defibrillator lead extraction: Predictors of mortality during follow-up. Pacing Clin Electrophysiol 33: 209-216, 2010. 